\title{
Participatory Design Methods to Define Educational Goals for Full-Body Interaction
}

\begin{abstract}
The paper presents an exploratory study aimed toward including children in the design of an Interactive Learning Environment based on Full-Body Interaction. The study proposes a method based on using Participatory Design techniques to analyze core meanings and misconceptions of children. The aim is to identify appropriate learning goals and to define concepts capable of bridging between children's knowledge and novel contents. Furthermore, it explores a novel approach aimed at fostering design methods suitable for a Full-Body Interaction experience. The results show the effectiveness of the method in defining guidelines during a first design iteration of a Learning Environment based on environmental education.
\end{abstract}

\section{Author Keywords}

Full-Body Interaction; Embodied Cognition; Participatory Design; Formative Assessment; Game Design; Narrative Inquiry

\section{ACM Classification Keywords}

H.5.1 Information Interfaces and Presentation (e.g., HCI): Multimedia Information Systems - Artificial, augmented, and virtual realities; User Interfaces -

\section{Evaluation/methodology}

\section{INTRODUCTION}

Recently, there has been an increasing interest in the potential of Full-Body Interaction as mediator of learning processes. Research in this field, based on the embodied cognition framework, suggests the hypothesis that physical activity and specific gesture may facilitate learning of abstract concepts through concrete experiences [4, 5, 6]. Starting from this perspective several Full-Body Interaction Learning Environments (FUBILEs) have been developed. However, previous research has failed to develop robust design methods for the development of effective learning

License: The author(s) retain copyright, but ACM receives an exclusive publication license. experiences.

A major shortcoming in this area has been found in the lack of research efforts aimed at including children's contributions in the design process. Promising possibilities can be found in Participatory Design (PD) methods aimed at incorporating children as informants or as co-designers in early stages of the design process [2]. However, two fundamental challenges have been identified in the use of PD for the development of FUBILEs. The first challenge is the need to define learning contents that are aligned with children's understanding and that are suitable for the features of Full-Body Interaction. As several researches point out, it is often difficult to combine the perspectives of children and experts since some proposals can be unworkable in computational terms or the preferences of children could actually come into conflict with the pedagogical goals [12]. Such difficulty is increased by the specific affordances of Full-Body Interaction, which requires a careful mapping between content and the embodied experience.

The second challenge refers to the need to develop methods that facilitate designing for and with the body. Most PD methods have been mainly developed to design desktopbased applications or similar products. However, for the design of FUBILEs some of these methods are not suitable since they do not take into account the specific features of body-based interaction or the configuration of spatial needs. Little information is available on the enquiry of bodilybased activities such as bodystorming to simulate interaction for games [7].

Hence, the emphasis of this paper is on exploring suitable design methods to develop effective FUBILEs. Such research is oriented toward defining appropriate learning goals and exploring methods for integrating PD and FullBody Interaction. For this purpose we conducted an explorative design study involving children through a playful PD approach. The aim of the workshop was to analyze children's previous knowledge in order to identify misconceptions that need to be addressed as educational requirements and concepts that can bridge between what the children already know and novel knowledge to be acquired by them. Furthermore, the use of bodily-based techniques has been explored in order to evaluate its effectiveness in facilitating design for Full-Body Interaction. 


\section{EXPLORATORY WORKSHOP}

The aim of the project was to develop a FUBILE to foster understanding specific issues of environmental education. In order to properly define learning goals the study was divided into two main parts: firstly, the identification of requirements by consulting experts in environmental education; secondly, the organization of a participatory workshop with children.

The goals of the workshop were to examine the mental models that children have around environmental education and to explore how to adapt PD methods for the development of FUBILEs.

In order to examine children's mental models we focus our study toward the analysis of their current knowledge. We embrace this by examining their misconceptions and their core meanings about the environment (i.e. what children consider to be relevant, what they select and remember). Such an approach was aimed at identifying knowledge gaps that need to be addressed as learning goals. Furthermore, the analysis was oriented toward defining concepts that can bridge between what children already know and novel knowledge, according to the method of guided participation proposed by Rogoff. In this instructional technique the author suggests the fundamental importance of using children's previous knowledge as an entry path for the comprehension of novel concepts [11].

On the other hand, to explore the possibilities of including bodily-based techniques that facilitate the design of experiences fitting within the features of Full-Body Interaction, we hypothesized that the inclusion of a bodilybased training would be beneficial. For this purpose, in the workshop we conducted a warming-up activity based on two different conditions: verbal- and bodily-based training. Our hypothesis was that children assigned to bodily-based training would enjoy more the PD and would propose design solutions which would be more appropriate for the features of Full-Body Interaction.

\section{METHOD AND PROCEDURE}

The exploratory study was carried out on three consecutive days in our Full-Body interaction laboratory. A total of 68 children between 10 and 12 years old participated. Each session had a duration of approximately $45 \mathrm{~min}$. Children were divided in groups of 4 to 5 members and the groups were randomly assigned to one of the two conditions (bodily- and verbal- based training).

Both conditions mainly differed in the warm up activities. The bodily-based training consisted in corporal exploration of the local space, pattern of movement and gesture coordination. Whereas, in the verbal-based training the children carried out activities based on speech exploration (i.e. constrains on the use of certain letters) and vocal coordination. Both methods were derived from theatrical practices.
After the warm up activity, we conducted a game based on the mechanics of the Pictionary game in both conditions. The provided words were related to environmental issues, such as solar energy or ecological footprint. The goal of the activity was twofold. On the one hand, we wanted to evaluate which terms the children were familiar with and their previous knowledge and misconceptions on environmental topics. On the other hand, the activity was useful to explore their representations of concrete concepts.

In the last part of the workshop, the children were asked to produce two lists of actions related to environmental issues classifying in positive or negative actions. After the brainstorming phase, the children were introduced to the next activity aimed toward facilitating the design of a FUBILE. The instructions were provided by incorporating a narrative inquiry [1] activity where we handed out a fictive letter of a young boy writing from the future. The letter was read aloud by one of the group members. Thus, the children were confronted with the critical situation of the environment in 100 years. Starting from this letter they were asked to invent a game based on body movement. The aim of this game was to make other people aware of strategies to avoid further degradation of nature and to improve our habits in relation to environmental issues. After a short explanation, the children produced drawings and written game descriptions. The research team supported the groups in the design process through verbal advice. Then, each team presented their design ideas to the entire group. To test the likability of the activities, the children were asked to fill out a questionnaire.

\section{EVALUATION METHODS}

Evaluation was oriented toward analyzing children's mental models and assessing the effects of the warm up activity on the unfolding of the workshop.

\section{Analyzing children's mental models}

The analysis of children's mental models was carried out using qualitative methods based on the analysis of the video recordings of the sessions, children's productions (i.e. drawings, lists, and written descriptions) and annotations.

After the workshop sessions, the outputs from each activity were examined. The analysis was oriented toward identifying children's previous knowledge, misconceptions and core meanings related to environmental education. Data from the workshop were analyzed as follows. First, the video transcription of the proposed games and text from the list were analyzed using the NVivo10 software. Video transcriptions were coded according to a grounded theory approach. From the data collected, key points were then marked with a series of codes which were extracted from the text. The codes were grouped into similar concepts in order to make them more workable and finally categories were formed. Additionally, we analyzed our observations and annotations of the activities individually. 


\section{Analysis of the effects of the warm up activities}

The evaluation of the effect of the warm up activities was based on two measures: suitability and capability. The term suitability refers to the likability of the activities and how much the children felt engaged and involved as active participants in the design process. On the other hand, capability represents the extent to which the activities can produce useful results for the design [9].

To assess suitability children were asked to rate the likability of the attended activities through a Likert-scale questionnaire ranging from 1 to 10 based on the local grading system with the value 10 as highest achievable grade. On the other hand, to evaluate capability a transcription of the proposed games was rated on a 0 to 5 scale by three experts blind to conditions in order to estimate the degree to which the proposed games fit with the specificities of Full-Body Interaction.

\section{RESULTS}

\section{Children's mental models}

By analyzing video recordings of the sessions, children's drawings and our annotations during the sessions, we obtained a better understanding of the previous knowledge and misconceptions of the children related to environmental issues. Using the grounded theory approach we identified that 14 out of the 26 proposed games were related to the topics of recycling or waste (see Figure 1) and 8 out of 26 referred to plants or trees. Examining the more frequent environmental locations of the proposed games, we found that 11 were related to natural settings, 4 to home settings and other 4 to urban settings.

Furthermore, this method gave us insights about the following misconceptions. Firstly, 9 out of 26 games wrongly applied the idea of "accumulation" to environmental aspects such as waste and watering plants. The typical game's mechanics of rewarding who obtains more items was therefore translated to "whoever obtains more waste or whoever gives more water to the plants, wins". Such proposals reveal an inadequate model, which does not take into account that reducing waste is a priority over recycling large amounts of waste. Also, many children believed that the player needs a reward for activities, which are good for the environment. Some also proposed positive actions (e.g. recycling, planting trees) as punishment activities for players who lose in the game (e.g. "if you lose you have to go to plant trees").

By analyzing observations and annotations we could detect more misconceptions. The concept of "light pollution" was incomprehensible; some children related it to the sun and they did not understand its effects on nature. The same occurred with the rather abstract concept of "contamination". Many children did not understand its meaning or related it only to rubbish.

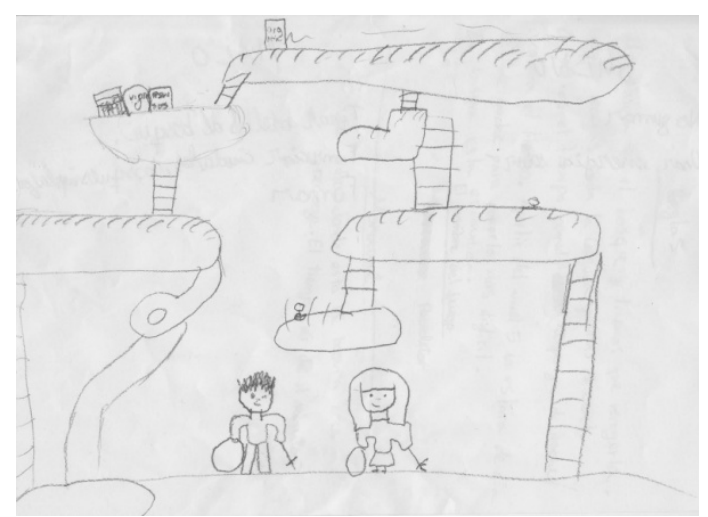

Figure 1. Game proposal based on collecting waste

\section{Suitability}

To analyze children's answers to the likeability questionnaire an independent t-test was performed between the two conditions: bodily-based and verbal-based training. Children who started the workshop with bodily-based activities $(\mathrm{M}=8.75, \mathrm{SD}=1.554)$ rated the activities significantly more positively than those with verbal-based activities $(\mathrm{M}=7.10, \mathrm{SD}=3.986), \mathrm{t}(32,794)=2.081, \mathrm{p}=$ .045 . This means that, although the narrative inquiry was the same in both conditions, children assigned to the bodilybased training liked it more than those assigned to the verbal-based training. Thus, we can conclude that the bodily-based training primed the children's perception of the overall workshop activities.

\section{Capability}

In order to assess the extent to which the proposed games fit with the specificities of Full-Body Interaction we firstly calculate the average between raters for each game. Subsequently an independent t-test was performed between the two conditions. No significant difference was found between the two conditions $(\mathrm{p}=0,752)$.

\section{DISCUSSION}

The method used to analyze children's mental model by focusing in core meanings and misconceptions showed to be highly effective to develop guidelines for the development of a first design iteration.

Main insights were related to the definition of area of knowledge that need to be addressed and to the identification of bridging concepts. Children have quite a clear idea about the local system of recycling and home practices related to saving energy or water. These topics should not be addressed directly since they already know them. However, these topics can be used to evoke some new concepts.

Balance, equilibrium and rhythm of nature is an important topic because 9 over 26 games presented misconceptions related to the notion of "the more the better", e.g. the children had difficulties to understand that reducing waste is more important than recycling. Potential topics to be addressed would be: to reduce contamination or generate as 
little as possible; the balance of ecosystems related to equilibrium between resources; or the meaning of contamination, its origin and global effects. Many topics around environment and abstract concepts such as light pollution or contamination are related only to home practice. We detected a lack of knowledge on global processes around environmental topics, such as the role of industries or specific nations. The children were also not aware of their own responsibility in this process and how their actions have an impact on all living beings on the planet, including themselves. From this analysis we redefined the learning goals and developed a first prototype aimed toward countering the lack of knowledge by focusing on an abstract concept such as an eco-system and its elements. Furthermore, to incorporate bridging concepts, we inserted elements such as waste reduction and plant growing in the Learning Environment.

On the other hand, results related to the effects of the warm up activity show that, despite bodily-based training fostered the overall engagement of the children during the workshop and primed their perception of the subsequent activities, it however did not facilitate the design of games which are more suitable for the features of Full-Body Interaction. Even if children engagement is fundamental for the success of PD workshops, further research is necessary in order to

\section{REFERENCES}

1. Dindler, C., \& Iversen, O. Fictional Inquiry -design collaboration in a shared narrative space. CoDesign 3, (4), (2007), 213-234.

2. Druin, A. Cooperative Inquiry : Developing New Technologies for Children with Children. In Human Factors in Computing Systems (CHI) 14(1999), 592599.

3. Druin, A. The role of children in the design of new technology. Behaviour and information technology 21(1), (2002), 1-25.

4. Freiler, T. J. Learning Through the Body. New Directions for Adults and Continuing Education 119 (2008), 37-48.

5. Glenberg, A. M. Embodiment as a unifying perspective for psychology. WIREs Cognitive Science1 (2010), 586-596.

6. Goldin-Meadow, S. Learning through gesture. Wiley Interdisciplinary Reviews. Cognitive Science 2(6) (2011), 595-607.

7. Hemmert, F., Hamann, S., Löwe, M., Zeipelt, J., \& Joost, G. Co-Designing with Children: A Comparison of explore how to adapt PD methods for Full-Body Interaction.

\section{CONCLUSION AND FUTURE WORK}

We have presented an exploratory study aimed toward including children in the design of an Interactive Learning Environment based on Full-Body Interaction. Our proposed method focuses on assessing children's mental models through the analysis of core meanings and misconceptions. Furthermore, we have undertaken a preliminary exploration of the possibilities of including body-based activities in PD.

Based on our experiences, we conclude that the method used to analyze children's mental model was very useful to identify learning goals and define bridging concepts between previous knowledge and novel concepts. This method facilitated formulating design guidelines for the development of a novel FUBILE. On the other hand, further research is necessary to deepen in the strategies for properly adapting PD methods for Full-Body Interaction.

\section{ACKNOWLEDGMENTS}

We thank all the participants from local schools for their time and motivation during the participatory workshop. We are also very grateful for the valuable information and materials about environmental education provided by \{removed for anonymity\}

Embodied and Disembodied Sketching Techniques in the Design of Child Age Communication Devices. In Proc. of the $9^{\text {th }}$ International Conference on Interaction (2010), 202-205..

8. Kelly, S. R., Mazzone, E., Horton, M., \& Read, J. C. Bluebells : A Design Method for Child-Centred Product Development. NordiCHI(2006).,14-18.

9. Mazzone, E., Tikkanen, R., Read, J. C., Iivari, N., \& Beale, R. Integrating children's contributions in the interaction design process. International Journal of Arts and Technology 5 (2012).

10. Nesset, V., \& Large, A. Children in the information technology design process: A review of theories and their applications. Library \& Information Science Research, 26(2) (2004), 140-161.

11. Rogoff, B. Apprenticeship in thinking: Cognitive development in social context. Oxford University Press New York, NY, USA, 1990.

12. Scaife, M., Rogers, Y. Kids as informants: telling us what we didn't know or confirming what we knew already? The design of children's technology. Morgan Kaufmann Publishers Inc (1998) 27-50. 